\title{
No-Scale Supergravity Realization of the Starobinsky Model of Inflation
}

\author{
John Ellis, ${ }^{1, *}$ Dimitri V. Nanopoulos, ${ }^{2, \dagger}$ and Keith A. Olive ${ }^{3, \neq}$ \\ ${ }^{1}$ Department of Physics, Theoretical Particle Physics and Cosmology Group, King's College London, \\ London WC2R 2LS, United Kingdom and Theory Division, CERN, CH-1211 Geneva 23, Switzerland \\ ${ }^{2}$ George P. and Cynthia W. Mitchell Institute for Fundamental Physics and Astronomy, Texas A\&M University, \\ College Station, Texas 77843, USA, Astroparticle Physics Group, Houston Advanced Research Center (HARC), \\ Mitchell Campus, Woodlands, Texas 77381, USA, and Division of Natural Sciences, Academy of Athens, \\ 28 Panepistimiou Avenue, Athens 10679, Greece \\ ${ }^{3}$ William I. Fine Theoretical Physics Institute, School of Physics and Astronomy, University of Minnesota, \\ Minneapolis, Minnesota 55455, USA \\ (Received 8 May 2013; published 9 September 2013; corrected 12 September 2013)
}

\begin{abstract}
We present a model for cosmological inflation based on a no-scale supergravity sector with an $\mathrm{SU}(2,1) / \mathrm{SU}(2) \times \mathrm{U}(1)$ Kähler potential, a single modulus $T$, and an inflaton superfield $\Phi$ described by a Wess-Zumino model with superpotential parameters $(\mu, \lambda)$. When $T$ is fixed, this model yields a scalar spectral index $n_{s}$ and a tensor-to-scalar ratio $r$ that are compatible with the Planck measurements for values of $\lambda \simeq \mu / 3 M_{P}$. For the specific choice $\lambda=\mu / 3 M_{P}$, the model is a no-scale supergravity realization of the $R+R^{2}$ Starobinsky model.
\end{abstract}

DOI: 10.1103/PhysRevLett.111.111301

The initial release of cosmic microwave background data from the Planck satellite [1] confronts theorists of cosmological inflation [2,3] with a challenge. On the one hand, the data have many important features that are predicted qualitatively by the inflationary paradigm. For example, there are no significant signs of non-Gaussian fluctuations or hints of nontrivial topological features such as cosmic strings, and the spectrum of scalar density perturbations exhibits a significant tilt: $n_{s} \simeq 0.960 \pm 0.007$, as would be expected if the effective scalar energy density decreased gradually during inflation. On the other hand, many previously popular field-theoretical models of inflation are ruled out by a combination of the constraint on $n_{s}$ and the tensor-to-scalar ratio $r<0.08$ as now imposed by Planck et al: see, e.g., Fig. 1 of [1]. The only model with truly successful predictions displayed in Fig. 1 of [1] is the $R^{2}$ inflation model of Starobinsky [4], though similar predictions are made in Higgs inflation [5] and related models [6].

In the following paragraphs we motivate the approach to inflation taken in this Letter, which casts a new light on the Starobinsky model [4] and embeds it in a more general theoretical context that connects with other ideas in particle physics. Specifically, the upper limit on $r$ implies that the energy scale during inflation must be much smaller than the Planck energy, $\sim 10^{19} \mathrm{GeV}$. Such a hierarchy of energy scales can be maintained naturally, without fine-tuning, in a theory with supersymmetry [7]. As is well known, (approximate) supersymmetry has many attractive features, such as providing a natural candidate for dark matter and facilitating grand unification, as well as alleviating the fine-tuning of the electroweak scale. In the context of early-universe cosmology, one must combine supersymmetry with gravity via a suitable supergravity
PACS numbers: 04.65.+e, 04.50.Kd, 12.60.Jv, 98.80.Cq

theory [8], which should accommodate an effective inflationary potential that varies slowly over a large range of inflaton field values. This occurs naturally in a particular class of supergravity models [9], which are called "no scale" because the scale at which supersymmetry is broken is undetermined in a first approximation, and the energy scale of the effective potential can be naturally much smaller than $\sim 10^{19} \mathrm{GeV}$, as required by the cosmic microwave background data. No-scale models have the additional attractive feature that they arise in generic four-dimensional reductions of string theory [10], though this does not play an essential role in our analysis. The attractive features of this no-scale supergravity framework for inflation do not depend sensitively on the supersymmetry-breaking scale, which could be anywhere between the experimental lower limit $\sim 1 \mathrm{TeV}$ from the $\mathrm{LHC}[11]$ and $\sim 10^{10} \mathrm{TeV}$ from the tensor-to-scalar ratio.

We now discuss these motivations at greater length before entering into the details of our inflationary model.

Since the energy scale during the inflationary epoch is typically $\ll M_{P}$, it is natural to study renormalizable models, i.e., some combination of $\phi^{2}, \phi^{3}$, and $\phi^{4}$ in the single-field case. In this spirit, it was shown in $[12,13]$ that a single-field model with a potential of the form

$$
V=A \phi^{2}(v-\phi)^{2}
$$

could easily produce Planck-compatible values of $\left(n_{s}, r\right)$ for a suitable number of $e$-folds before the end of inflation $N \sim 50-60$. This simple symmetry-breaking potential has a long pedigree, having been proposed initially in [14] (for a review, see [2]), where it was argued that successful inflation would require a small value of $A$ and $v>M_{P}$. 
As we pointed out in [7], in addition to all the wellknown reasons for postulating low-scale supersymmetry, the small values of the quartic and quadratic couplings that would be required in a successful inflationary model, e.g., $A$ in the above example, become technically natural in the presence of low-scale supersymmetry. In particular, small values of $\delta \rho / \rho$ become technically natural if approximate supersymmetry is invoked [7], and if the grand unified theory Higgs boson is distinguished from the singlet field that produces inflation, that later became known as the inflaton [15].

The simplest globally supersymmetric model is the Wess-Zumino model with a single chiral superfield $\Phi$ [16], which is characterized by a mass term $\hat{\mu}$ and a trilinear coupling $\lambda$, with the superpotential

$$
W=\frac{\hat{\mu}}{2} \Phi^{2}-\frac{\lambda}{3} \Phi^{3}
$$

As was discussed in [13], the effective potential of the Wess-Zumino model reduces to (1) when the imaginary part of the scalar component of $\Phi$ vanishes, in which case this model yields Planck-compatible inflation for a suitable small value of $\lambda$.

However, global symmetry is not enough. As discussed above, in the context of early-universe cosmology one should certainly include gravity and hence construct a locally supersymmetric model, i.e., upgrade to supergravity [8]. The first attempt at constructing an inflationary model in $N=1$ supergravity proposed a generic form for the superpotential for a single inflaton [17], the simplest form being $W=m^{2}(1-a \Phi)^{2}$ [18]. As discussed in [19], while this relatively simple model is capable of sufficient inflation, it is an example of accidental inflation in the sense that the coefficient of the linear term in the superpotential $a$ must be extremely close to unity. This model has also become one of Planck's casualties. The scalar-totensor ratio in this model is very small, but the value of $n_{s}$ predicted in this model is $n_{s} \simeq 1-4 / N=0.933$ for $N=60$ [20], since the effective potential varies insufficiently slowly.

In a supergravity model with a generic Kähler potential for the chiral supermultiplets there are quadratic $|\phi|^{2}$ terms, which cause variations in the effective potential that destroy its suitability for inflation, an obstacle known as the $\eta$ problem [3]. As was pointed out in [21], a natural solution to this problem is offered by no-scale supergravity [9], whose motivations were summarized earlier. In such a model, quadratic terms are suppressed, and the effective scalar potential resembles that in a globally supersymmetric model, thanks to an underlying noncompact $\mathrm{SU}(N, 1) / \mathrm{SU}(N) \times \mathrm{U}(1)$ symmetry.

Other no-scale supergravity approaches have also been proposed [22], as well as models based on a noncompact Heisenberg symmetry [23], a shift symmetry [24-26], or string theory [27]. The $\mathrm{SU}(N, 1)$ model [21] was based on the superpotential $W=m^{2}\left(\phi-\phi^{4} / 4\right)$ and gives similar predictions for the inflationary parameters as the minimal $N=1$ model discussed above. This too is an example of accidental inflation [19], and a small change in the coefficient of the quartic term would lead to parameters consistent with Planck data [1].

In this Letter we show how one can elevate the simplest globally supersymmetric Wess-Zumino inflationary model of [13] to a no-scale supergravity version (NSWZ). Concretely, we study a model in which the inflaton superfield is embedded in an $\mathrm{SU}(2,1) / \mathrm{SU}(2) \times \mathrm{U}(1)$ no-scale supergravity sector together with a modulus field $T$ (which we assume to be fixed by other dynamics [28]) and find a range of the parameters where it is compatible with the Planck data [1]. Quite remarkably, as we show, the NSWZ model is the conformal equivalent of an $R+R^{2}$ model of gravity for one specific value of $\hat{\mu} / \lambda$, so that in this case our realization of inflation in the NSWZ model is equivalent to the Starobinsky model of inflation [4]. Thus, we embed this model in a broader and attractive theoretical framework.

We first recall the basic relevant formulas governing the kinetic term and the effective potential of scalar fields $\phi$ in $\mathcal{N}=1$ supergravity, specializing to the no-scale case with noncompact $\mathrm{SU}(N, 1) / \mathrm{SU}(N) \times \mathrm{U}(1)$ symmetry. The scalar sector may be characterized in general by a Hermitian Kähler function $K$ and a holomorphic superpotential $W$ via the combination $G \equiv K+\ln W+\ln W^{*}$. The kinetic term is then given by $K_{i}^{j^{*}} \partial_{\mu} \phi^{i} \partial \phi_{j}^{*}$, where the Kähler metric $K_{i}^{j^{*}} \equiv \partial^{2} K / \partial \phi^{i} \partial \phi_{j}^{*}$, and the effective potential is

$$
V=e^{G}\left[\frac{\partial G}{\partial \phi^{i}} K_{j^{*}}^{i} \frac{\partial G}{\partial \phi_{j}^{*}}-3\right]
$$

where $K_{j^{*}}^{i}$ is the inverse of the Kähler metric $K_{i}^{j^{*}}$.

In the minimal no-scale $\mathrm{SU}(2,1) / \mathrm{SU}(2) \times \mathrm{U}(1)$ case, there are two complex scalar fields: $T$, a modulus field, and $\phi$, which we identify as the inflaton field, with the Kähler function $K=-3 \ln \left(T+T^{*}-|\phi|^{2} / 3\right)$. In this case, the kinetic terms for the scalar fields $T$ and $\phi$ become

$$
\begin{aligned}
\mathcal{L}_{K E}= & \left(\partial_{\mu} \phi^{*}, \partial_{\mu} T^{*}\right)\left(\frac{3}{\left(T+T^{*}-|\phi|^{2} / 3\right)^{2}}\right) \\
& \times\left(\begin{array}{cc}
\left(T+T^{*}\right) / 3 & -\phi / 3 \\
-\phi^{*} / 3 & 1
\end{array}\right)\left(\begin{array}{c}
\partial^{\mu} \phi \\
\partial^{\mu} T
\end{array}\right),
\end{aligned}
$$

and the effective potential becomes

$$
V=\frac{\hat{V}}{\left(T+T^{*}-|\phi|^{2} / 3\right)^{2}}: \hat{V} \equiv\left|\frac{\partial W}{\partial \phi}\right|^{2} .
$$

In early no-scale models [21,23] it was assumed that $K$ was fixed so that the potential up to a rescaling was simply $\hat{V}$. Here we assume that the $T$ field has a vacuum expectation value $(\mathrm{VEV}) 2\langle\operatorname{Re} T\rangle=c$ and $\langle\operatorname{Im} T\rangle=0$ that is determined by nonperturbative high-scale dynamics [28], as in the Kähler correction provided in [29]. In this case, we may 
neglect the kinetic mixing between the $T$ and $\phi$ fields in (4), and are left with the following effective Lagrangian for the inflaton field $\phi$ :

$$
\mathcal{L}_{\text {eff }}=\frac{c}{\left(c-|\phi|^{2} / 3\right)^{2}}\left|\partial_{\mu} \phi\right|^{2}-\frac{\hat{V}}{\left(c-|\phi|^{2} / 3\right)^{2}} .
$$

We assume as in [13] the minimal Wess-Zumino superpotential (2) for the inflaton field.

To better study the potential for the inflaton, we first transform $\phi$ to the field $\chi$ :

$$
\phi=\sqrt{3 c} \tanh \left(\frac{\chi}{\sqrt{3}}\right) .
$$

With this field redefinition, the Lagrangian becomes

$$
\begin{aligned}
\mathcal{L}_{\mathrm{eff}}= & \operatorname{sech}^{2}\left[\left(\chi-\chi^{*}\right) / \sqrt{3}\right]\left[\left|\partial_{\mu} \chi\right|^{2}-\left(\frac{3}{c}\right) \mid \sinh (\chi / \sqrt{3})\right. \\
& \left.\times\left.[\hat{\mu} \cosh (\chi / \sqrt{3})-\sqrt{3 c} \lambda \sinh (\chi / \sqrt{3})]\right|^{2}\right] .
\end{aligned}
$$

Clearly the VEV of the $T$ field can be absorbed into the definition of the mass and, writing $\hat{\mu}=\mu \sqrt{c / 3}$, the potential becomes

$V=\mu^{2}\left|\sinh (\chi / \sqrt{3})\left(\cosh (\chi / \sqrt{3})-\frac{3 \lambda}{\mu} \sinh (\chi / \sqrt{3})\right)\right|^{2}$.

Writing $\chi$ in terms of its real and imaginary parts: $\chi=$ $(x+i y) / \sqrt{2}$, and, for reasons which will become clear, considering the specific case where the quartic coupling $\lambda=\mu / 3$ (in Planck units), we have

$$
\begin{aligned}
\mathcal{L}_{\text {eff }}= & \frac{1}{2} \sec ^{2}(\sqrt{2 / 3} y)\left[\left(\partial_{\mu} x\right)^{2}+\left(\partial_{\mu} y\right)^{2}\right] \\
& -\mu^{2} \frac{e^{-\sqrt{2 / 3} x}}{2} \sec ^{2}(\sqrt{2 / 3} y)(\cosh \sqrt{2 / 3} x \\
& -\cos \sqrt{2 / 3} y) .
\end{aligned}
$$

The imaginary part of the inflaton is fixed to $y=0$ by the potential, having a mass $m_{y}=\mu / \sqrt{3}$ during inflation when $x$ is large and $m_{y}=\mu / \sqrt{6}$ at the end of inflation when $x=0$. Thus we expand the Lagrangian about $y=0$, in which case we have minimal kinetic terms for $x$ and $y$, accompanied by derivative interaction terms. The potential for the real part of the inflaton now takes the form

$$
V=\mu^{2} e^{-\sqrt{2 / 3} x} \sinh ^{2}(x / \sqrt{6}) .
$$

This potential is depicted in Fig. 1, where we also display the potential for values of $\lambda$ slightly perturbed from the nominal value of $\mu / 3$.

We use the standard slow-roll expressions for the tensorto-scalar ratio $r$ and the spectral index $n_{s}$ for the scalar perturbations in terms of the slow-roll inflation parameters $\epsilon, \eta[3]$, which we evaluate in terms of the canonically normalized field $x$. In the NSWZ model described above, the VEV of $T$ is absorbed in the definition of the mass parameter $\mu$, which is determined by the normalization of the quadrupole. For the special case $\lambda=\mu / 3$, we have

$$
A_{s}=\frac{V}{24 \pi^{2} \epsilon}=\frac{\mu^{2}}{8 \pi^{2}} \sinh ^{4}(x / \sqrt{6}),
$$

implying a value $\mu=2.2 \times 10^{-5}$ in Planck units for $N=55: \mu$ varies between $(1.8-3.4) \times 10^{-5}$ over the range of NSWZ models considered here. Setting the remaining NSWZ parameter $\lambda=\mu / 3$, we have

$$
\epsilon=\frac{1}{3} \operatorname{csch}^{2}(x / \sqrt{6}) e^{-\sqrt{2 / 3} x},
$$

$$
\eta=\frac{1}{3} \operatorname{csch}^{2}(x / \sqrt{6})\left(2 e^{-\sqrt{2 / 3} x}-1\right),
$$

which allows us to determine the quantities $\left(n_{s}, r\right)$, once the value of the field $x$ is fixed by requiring $N=50-60$ $e$-folds. The nominal choice of $N=55$ yields $x=5.35$, $n_{s}=0.965$, and $r=0.0035$.

Figure 2 displays the predictions for $\left(n_{s}, r\right)$ of the NSWZ model for five choices of the coupling $\lambda$ that yield $n_{s} \in$ $[0.93,1.00]$ and $N \in[50,60]$. The last 50-60e-folds of inflation arise as $x$ rolls to zero from $\sim 5.1-5.8$, the exact value depending on $\lambda$ and $N$. As one can see, the values of $\lambda$ are constrained to be close to the critical value $\mu / 3$, for which we find extremely good agreement with the Planck determination of $n_{s}$. The values of $r$ are rather small for $\lambda=\mu / 3$, varying over the range $0.0012-0.0084$, in the models considered.

At first sight, this success might appear to be another example of accidental inflation [19], but, as we now show, this choice of $\lambda$ has a more profound geometric interpretation. The alert reader may have noticed resemblances of both the potential shown in Fig. 1 and the values of $\left(n_{s}, r\right)$ found for the $\lambda=\mu / 3$ model with results for inflation in the $R+R^{2}$ model proposed by Starobinsky [4]. To further probe this resemblance, we examine the generalization of the Einstein-Hilbert action to contain an $R^{2}$ contribution, where $R$ is the scalar curvature,

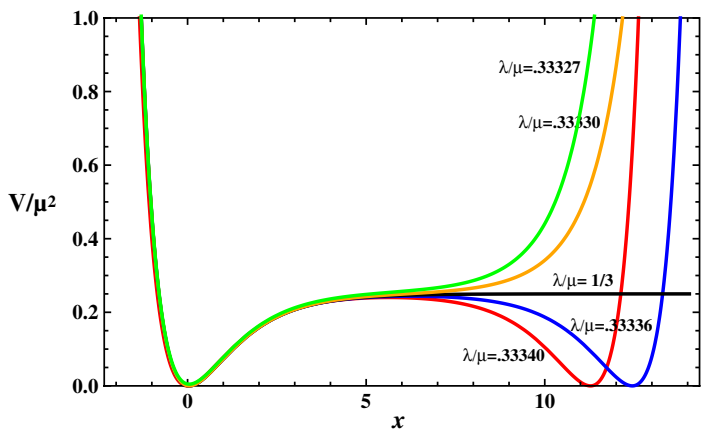

FIG. 1 (color online). The potential $V$ in the NSWZ model for choices of $\lambda \sim \mu / 3$ in Planck units, as indicated. 


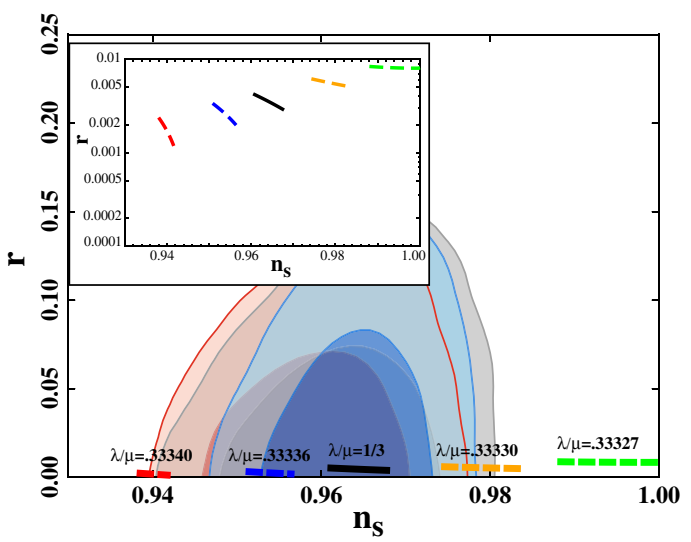

FIG. 2 (color online). Predictions from the NSWZ model for the tilt $n_{s}$ in the spectral index of scalar perturbations and for the tensor-to-scalar ratio $r$, compared with the $68 \%$ and $95 \%$ C.L. regions found in analyses of Planck and other data [1]. In the main panel the lines are labeled by the values of $\lambda / \mu$ (in Planck units) assumed in each case. In the inset, the same cases are shown on a $\log$ scale to better display the values of $r$.

$$
S=\frac{1}{2} \int d^{4} x \sqrt{-g}\left(R+R^{2} / 6 M^{2}\right),
$$

where $M \ll M_{P}$ is some mass scale. This theory is conformally equivalent to canonical gravity plus a scalar field $\varphi$ [30]. Making the transformation $\tilde{g}_{\mu \nu}=\left(1+\varphi / 3 M^{2}\right) g_{\mu \nu}$ and the field redefinition $\varphi^{\prime}=\sqrt{3 / 2} \ln \left[1+\left(\varphi / 3 M^{2}\right)\right]$, we obtain the action

$S=\frac{1}{2} \int d^{4} x \sqrt{-\tilde{g}}\left[\tilde{R}+\left(\partial_{\mu} \varphi^{\prime}\right)^{2}-\frac{3}{2} M^{2}\left(1-e^{-\sqrt{2 / 3} \varphi^{\prime}}\right)^{2}\right]$,

corresponding to a potential

$$
V=\frac{3}{4} M^{2}\left(1-e^{-\sqrt{2 / 3} \varphi^{\prime}}\right)^{2} .
$$

The potential (17) is identical with the potential (11) along the real direction of the NSWZ model. Moreover, we have the identification $M^{2}=\mu^{2} / 3$, which equals $\hat{\mu}^{2}$ for $c=\left\langle\left(T+T^{*}\right)\right\rangle=1$. Thus the Starobinsky mass $M$ is directly related to the NSWZ mass $\hat{\mu}$ in the superpotential (2). We note that similar potentials are also obtained in Higgs inflation and related models [5].

We have shown in this Letter that the simplest $\mathrm{SU}(2,1) / \mathrm{SU}(2) \times \mathrm{U}(1)$ no-scale supergravity model with a single modulus field $T$ and a single matter field $\phi$ with the simplest renormalizable Wess-Zumino superpotential, identified with the inflaton, is capable of yielding cosmological inflation with values of the scalar spectral tilt $n_{s}$ and the tensor-to-scalar ratio $r$ within the region favored by Planck and other data at the $68 \%$ C.L. Successful inflation is obtained for $\lambda \simeq \mu / 3$ in Planck units. This NSWZ model is a proof of the existence of acceptable models of inflation based on no-scale supergravity, and normally we would not advocate that its details should necessarily be taken literally. For example, a realistic no-scale model derived from a generic compactification of string theory would have more moduli fields, with many matter fields that could be the inflaton, with a superpotential more complicated than assumed here.

However, it is truly striking that the NSWZ model is conformally equivalent to the Starobinsky $R^{2}$ model [4] for the specific choice $\lambda=\mu / 3$ in Planck units. This correspondence suggests that there is a profound geometric interpretation of this model that remains to be understood.

J.E. thanks Djuna Croon, Mirjam Cvetič, Nick Mavromatos, Henry Tye, and Gary Shiu for discussions, and K. A. O. thanks Andrei Linde and Misha Voloshin for discussions. We thank A. Kehagias for pointing out to us a previous derivation of the $R+R^{2}$ model from a different version of no-scale $\mathrm{SU}(2,1) / \mathrm{SU}(2) \times \mathrm{U}(1)$ supergravity [31]. The work of J.E. was supported in part by the London Centre for Terauniverse Studies (LCTS), using funding from the European Research Council via the Advanced Investigator Grant No. 267352. The work of D. V.N. was supported in part by the DOE Grant No. DE-FG03-95-Er-40917. The work of K. A. O. was supported in part by DOE Grant No. DE-FG02-94ER40823 at the University of Minnesota.

*John.Ellis@cern.ch

†dimitri@physics.tamu.edu

olive@physics.umn.edu

[1] P. A. R. Ade et al. (Planck Collaboration), arXiv: 1303.5082.

[2] K. A. Olive, Phys. Rep. 190, 307 (1990).

[3] See, for example, A.D. Linde, Particle Physics and Inflationary Cosmology (Harwood, Chur, Switzerland, 1990); D. H. Lyth and A. Riotto, Phys. Rep. 314, 1 (1999); J. Martin, C. Ringeval, and V. Vennin, arXiv: 1303.3787.

[4] A. A. Starobinsky, Phys. Lett. 91B, 99 (1980); V.F. Mukhanov and G. V. Chibisov, Pis'ma Zh. Eksp. Teor. Fiz. 33, 549 (1981) [JETP Lett. 33, 532 (1981)]; A. A. Starobinsky, Sov. Astron. Lett. 9, 302 (1983).

[5] F. Bezrukov and M. Shaposhnikov, J. High Energy Phys. 07 (2009) 089.

[6] A. Linde, M. Noorbala, and A. Westphal, J. Cosmol. Astropart. Phys. 03 (2011) 013; S. Ferrara, R. Kallosh, A. Linde, A. Marrani, and A. Van Proeyen, Phys. Rev. D 83, 025008 (2011).

[7] J.R. Ellis, D. V. Nanopoulos, K. A. Olive, and K. Tamvakis, Phys. Lett. 118B, 335 (1982); Phys. Lett. 120B, 331 (1983); Nucl. Phys. B221, 524 (1983).

[8] D.Z. Freedman, P. van Nieuwenhuizen, and S. Ferrara, Phys. Rev. D 13, 3214 (1976); S. Deser and B. Zumino, Phys. Lett. 62B, 335 (1976).

[9] E. Cremmer, S. Ferrara, C. Kounnas, and D. V. Nanopoulos, Phys. Lett. 133B, 61 (1983); J. R. Ellis, C. Kounnas, and D. V. Nanopoulos, Nucl. Phys. B247, 373 
(1984); A. B. Lahanas and D. V. Nanopoulos, Phys. Rep. 145, 1 (1987).

[10] E. Witten, Phys. Lett. 155B, 151 (1985).

[11] O. Buchmueller, R. Cavanaugh, M. Citron, A. De Roeck, M. J. Dolan, J. R. Ellis, H. Flacher, S. Heinemeyer et al., Eur. Phys. J. C 72, 2243 (2012).

[12] R. Kallosh and A. D. Linde, J. Cosmol. Astropart. Phys. 04 (2007) 017.

[13] D. Croon, J. Ellis, and N. E. Mavromatos, Phys. Lett. B 724, 165 (2013).

[14] A. D. Linde, Phys. Lett. 132B, 317 (1983); Pis'ma Zh. Eksp. Teor. Fiz. 37, 606 (1983) [JETP Lett. 37, 724 (1983)]; A. Albrecht and R.H. Brandenberger, Phys. Rev. D 31, 1225 (1985).

[15] D. V. Nanopoulos, K. A. Olive, and M. Srednicki, Phys. Lett. 127B, 30 (1983).

[16] J. Wess and B. Zumino, Nucl. Phys. B70, 39 (1974).

[17] D. V. Nanopoulos, K. A. Olive, M. Srednicki, and K. Tamvakis, Phys. Lett. 123B, 41 (1983).

[18] R. Holman, P. Ramond, and G. G. Ross, Phys. Lett. 137B, 343 (1984).

[19] A. D. Linde and A. Westphal, J. Cosmol. Astropart. Phys. 03 (2008) 005.

[20] This model still falls within the region allowed by Planck at the $95 \%$ C.L. for $N=70$ or a slight deviation in $a$ from unity, by 1 part in $10^{6}$.

[21] J. R. Ellis, K. Enqvist, D. V. Nanopoulos, K. A. Olive, and M. Srednicki, Phys. Lett. 152B, 175 (1985); 156B, 452(E) (1985).
[22] A.S. Goncharov and A.D. Linde, Classical Quantum Gravity 1, L75 (1984).

[23] P. Binetruy and M. K. Gaillard, Phys. Lett. B 195, 382 (1987); H. Murayama, H. Suzuki, T. Yanagida, and J. Yokoyama, Phys. Rev. D 50, R2356 (1994); S. Antusch, M. Bastero-Gil, K. Dutta, S. F. King, and P. M. Kostka, Phys. Lett. B 679, 428 (2009).

[24] M. Kawasaki, M. Yamaguchi, and T. Yanagida, Phys. Rev. Lett. 85, 3572 (2000); K. Nakayama, F. Takahashi, and T. T. Yanagida, Phys. Lett. B 725, 111 (2013).

[25] S. C. Davis and M. Postma, J. Cosmol. Astropart. Phys. 03 (2008) 015.

[26] R. Kallosh and A. Linde, J. Cosmol. Astropart. Phys. 11 (2010) 011; R. Kallosh, A. Linde, and T. Rube, Phys. Rev. D 83, 043507 (2011); R. Kallosh, A. Linde, K. A. Olive, and T. Rube, Phys. Rev. D 84, 083519 (2011).

[27] E. Silverstein and A. Westphal, Phys. Rev. D 78, 106003 (2008); L. McAllister, E. Silverstein, and A. Westphal, Phys. Rev. D 82, 046003 (2010); X. Dong, B. Horn, E. Silverstein, and A. Westphal, Phys. Rev. D 84, 026011 (2011).

[28] For examples showing that this is possible while preserving the no-scale structure and reducing to our model in a suitable limit, and hence justifying our subsequent analysis, see J. Ellis, D. V. Nanopoulos, and K. A. Olive, arXiv:1307.3537.

[29] J. R. Ellis, C. Kounnas, and D. V. Nanopoulos, Phys. Lett. 143B, 410 (1984).

[30] B. Whitt, Phys. Lett. 145B, 176 (1984).

[31] S. Cecotti, Phys. Lett. B 190, 86 (1987). 